\title{
Aumento do Risco Cardiovascular em Mulheres com Síndrome Metabólica
}

\section{Cardiovascular Risk in women with Metabolic Syndrome}

\author{
Rosimere J. Teixeira ${ }^{1}$ \\ Ana Borba Leite ${ }^{2}$ \\ Clarice A.S.A. Farias ${ }^{2}$ \\ Cíntia R. Sousa ${ }^{2}$ \\ Adryana M. Yugue ${ }^{2}$ \\ Aline A. Aguiar ${ }^{2}$ \\ Denise Ginzbarg ${ }^{3}$ \\ Maria Inez A. Padula ${ }^{4}$
}

\begin{abstract}
Resumo
A síndrome metabólica (SM) tem-se tornado uma epidemia, e isso representa um problema de saúde pública, mas seu verdadeiro papel como fator de risco cardiovascular - RCV - ainda é controverso. Nosso objetivo foi descrever o RCV em 45 mulheres com SM ( $n=25,51 \pm 12$ anos) e sem SM ( $n=20,36 \pm 13$ anos). Foram avaliados: IMC ; cintura abdominal; pressão arterial sistólica e diastólica (PAS e PAD); níveis de glicose (G) e insulina de jejum; G pós-prandial (Gpp); triglicerídeos; colesterol HDL e LDL. A sensibilidade à insulina foi avaliada pelo método de Homa-IR. A SM foi definida segundo o Programa Nacional de Educação em Colesterol. O RCV foi calculado pelo escore de Framingham e classificado como baixo, médio e alto. A cintura e a PAS foram maiores na SM, mas sem diferença quando relacionadas ao IMC e à PAD. Os níveis de G, Gpp e triglicerídeos foram maiores, enquanto o HDL foi menor na SM. O número de componentes, o RCV e a proporção de casos com médios ou altos RCV foram maiores na SM. O escore de risco se correlacionou com o número de componentes da síndrome metabólica - cSM - $(\mathrm{r}=0,62)$, cintura $(\mathrm{r}=0,45), \mathrm{G}$ $(\mathrm{r}=0,72)$, PAS ( $\mathrm{r}=0,59)$ e HDL ( $\mathrm{r}=-0,31)$, mas, na regressão múltipla, apenas com a $\mathrm{G}$ e a PAS $(\mathrm{r} 2=0,67, \mathrm{p}<0,007)$. O HOMA-IR mostrou correlação com a cintura $(\mathrm{r}=0,30, \mathrm{p}=0,04)$. A cintura abdominal é um bom marcador clínico da RI. As mulheres com SM apresentam maior risco para eventos cardiovasculares. Intervenções primárias dirigidas para os componentes da síndrome podem prevenir e reduzir a incidência de eventos cardiovasculares nessas mulheres.
\end{abstract}

Palavras-chave: Resistência à Insulina;

Síndrome Metabólica; Saúde da Mulher.
Key Words: Insulin Resistance; Metabolic Syndrome;

Women Health.

${ }^{1}$ Doutora em Endocrinologia pela UFRJ. Médica, Departamento de Medicina Integral, Familiar e Comunitária, Hospital Universitário Pedro Ernesto, Universidade do Estado do Rio de Janeiro, Rio de Janeiro, Brasil.

${ }^{2}$ Interna de Medicina, Faculdade de Ciências Médicas, Universidade do Estado do Rio de Janeiro, Rio de Janeiro, Brasil.

${ }^{3}$ Mestre em Endocrinologia, Universidade do Estado do Rio de Janeiro, Rio de Janeiro, Brasil.

${ }^{4}$ Doutora em Saúde Coletiva. Professora Adjunta, Departamento de Medicina Integral, Familiar e Comunitária, Hospital Universitário Pedro Ernesto, Universidade do Estado do Rio de Janeiro, Rio de Janeiro, Brasil. 


\begin{abstract}
Today, the metabolic syndrome (MS) epidemic turned into a public health problem. The true role of MS as a risk factor of cardiovascular disease however is not defined yet. Our objective was to describe the cardiovascular risk (CVR) in 45 women with $(n=25$, $51 \pm 12$ years) and without MS ( $n=20,36 \pm 13$ years). BMI, abdominal circumference and systolic and diastolic blood pressure (SBP and DBP) were evaluated. The levels of fasting glucose $(G)$ and insulin, post-prandial $G(p p G)$, triglycerides, cholesterol, HDL and $L D L$ were measured. Insulin sensibility was evaluated using the IRHoma method. SM was defined according to the National Cholesterol Education Program. The CVR was calculated according to the Framingham score and classified as low, medium and high. The abdominal circumference and SBP were significantly higher in SM, but there were no differences in relation to the BMI and DBP. G, Gpp and triglyceride levels were significantly bigher, while the HDL was lower in MS. The number of components, $C V R$ and the proportion of cases with medium or high risk were higher in the MS. $C V R$ was correlated with the number of components $(r=0,62)$, circumference $(r=0,45), G(r=0,72), \operatorname{SBP}(r=0,59)$ and HDL $(r=-0,31)$ and in the multiple regression analysis only with $G$ and $\operatorname{SBP}(r 2=0,67, p<0,007)$. HOMA-IR was correlated with the circumference $(r=0,30, p=0,04)$. Abdominal circumference is a good marker of IR. The women with MS are at a bigher risk of suffering cardiovascular events. We believe that primary care interventions directed to the components of the syndrome can prevent and reduce the incidence of $C V R$ in these women.
\end{abstract}

\section{Introdução}

Com os avanços tecnológicos e o controle das doenças infecto-contagiosas, houve aumento significativo na sobrevida, mas isso não foi sem ônus. O "moderno" estilo de vida, caracterizado por hábitos alimentares calóricos, por dietas ricas em gordura e pobres em fibras, associado ao sedentarismo, uso abusivo de álcool, tabagismo e estresse, tem papel importante na epidemia de obesidade ${ }^{1}$. A obesidade é frequentemente um problema que aflige parte das mulheres durante toda a vida. Postula- se que flutuações hormonais associadas com adolescência, gravidez, lactação e menopausa estariam correlacionadas com o aumento de risco de obesidade entre as mulheres ${ }^{2}$. A saúde geral da mulher é comprometida pela obesidade. Há doenças que afetam particularmente a mulher obesa, destacando-se a diminuição da fertilidade e o aumento da morbidade materna e fetal durante a gestação ${ }^{2}$. Por outro lado, algumas doenças associadas à obesidade podem estar presentes em ambos os sexos, como diabetes mellitus tipo 2 (DM2), hipertensão arterial (HA) e dislipidemia. Essas desordens fazem parte da Síndrome Metabólica (SM), que representa um dos maiores problemas de saúde pública da atualidade ${ }^{1}$. A SM é representada por um conjunto de fatores de risco para doença cardiovascular (DCV) e diabetes, muitos dos quais compartilham também resistência à insulina (RI) e obesidade. É importante destacar que a SM está associada a um aumento da mortalidade geral e da cardiovascular em torno de 1,5 a 2,5 vezes, respectivamen$\mathrm{te}^{3,4}$.

Reaven ${ }^{5}$ foi o primeiro a descrever a síndrome com o termo "síndrome X" para representar uma constelação de fatores de riscos associados à RI, tendo estabelecido a hipótese de que a insulina ou a ação da insulina pode ser um fator comum na etiologia dessas desordens. A obesidade foi descrita como um fator exacerbante, mas sem o mesmo significado fisiopatológico da RI.

Os fatores de risco da SM são de origem metabólica e consistem de dislipidemia aterogênica, elevação da pressão arterial, hiperglicemia, estado pró-trombótico e estado pró-inflamatório. A dislipidemia aterogênica compreende a elevação de lipoproteínas contendo apolipoproteína $\mathrm{B}$, hipertrigliceridemia, aumento da lipoproteína de baixa densidade (LDL) pequena e densa e baixos níveis de lipoproteína de alta densidade (HDL). A elevação dos níveis de glicose varia entre o pré-diabetes e o diabetes. $\mathrm{O}$ estado pró-trombótico significa anomalias nos fatores procoagulantes (isto é, aumento do fibrinogênio e fator VII), fatores anti-fibrinolíticos (isto é, plaminogen activator inbibidor type 1 PAI-1), aberrações plaquetárias e disfunção endotelial. $O$ estado pró-inflamatório é caracterizado pela elevação de 
citocinas circulantes e reagentes de fase aguda (isto é, proteína C reativa). Vários outros fatores de risco associados à RI vêm sendo incorporados, como a obesidade, especialmente a deposição de gordura visceral, hiperuricemia, microalbuminúria, angina microvascular e esteatose hepática nãoalcoólica como componentes da SM mais ampliada ${ }^{6,7,8}$. A obesidade e a RI também estão associadas a um alto risco para o desenvolvimento de Pubarca Precoce e Síndrome dos Ovários Policísticos (SOP) nas mulheres. Essas meninas, adolescentes e mulheres jovens apresentam vários achados típicos da SM - obesidade, hiperinsulinemia e alterações do perfil lipídico - desde a infância até a vida adulta, por isso tem sido sugerido que essas desordens também devem fazer parte da lista de componentes da SM9 9

É preocupante o fato de estas desordens estarem se tornando cada vez mais freqüentes nas populações. Os estudos em diferentes populações revelam prevalências elevadas da SM, sendo que as taxas são maiores nas mulheres $(10,7-40,5 \%)$ do que nos homens $(12,4-28,5 \%)^{10}$. Além da elevada prevalência, suas complicações crônicas - coronariopatia, acidente vascular cerebral, dentre outras - comprometem a qualidade de vida e a sobrevida da população. A abordagem precoce é de importância vital tanto para os clínicos quanto para os pediatras, que têm a responsabilidade do atendimento da maioria da população nos ambulatórios de cuidados primários. Nosso objetivo foi determinar o risco cardiovascular em mulheres com e sem SM.

\section{Casuística e Métodos}

Trata-se de um estudo retrospectivo, de corte transversal no qual foram avaliados os prontuários de 45 mulheres, entre 22 a 71 anos de idade, em acompanhamento no ambulatório de Medicina Integral do HUPE. O trabalho foi aprovado pelo Comitê de Ética em Pesquisa.

Foram levantados dados referentes aos parâmetros antropométricos: altura $(\mathrm{cm})$, peso $(\mathrm{kg})$ e medida da cintura abdominal $(\mathrm{cm})$. Para a medida da cintura abdominal foi considerado o ponto médio entre a crista ilíaca lateral e a costela inferior, com o paciente em posição supina e em expiração. $\mathrm{O}$ índice de massa corporal $\left(\mathrm{IMC}=\mathrm{kg} / \mathrm{m}^{2}\right)$ foi calculado, sendo considerado como obesidade o IMC = $30 \mathrm{~kg} / \mathrm{m}^{2}$ e como obesidade central a cintura abdominal > $88 \mathrm{~cm}$. A medida da pressão arterial foi aferida pela técnica auscultatória, preferencialmente após repouso, com as pacientes sentadas e de acordo com os procedimentos padronizados.

As amostras de sangue foram colhidas após dieta livre e jejum de 8 a 12 horas. A glicemia pós-sobrecarga de duas horas (Gpp - com 75 gramas de glicose VO) foi realizada nos pacientes com glicemia de jejum alterada (12 com SM e 10 sem SM). Foram utilizados os métodos GOD-PAP para a dosagem de glicose $(\mathrm{G}, \mathrm{VR}=70-110$ $\mathrm{mg} / \mathrm{dL}$ ) e o colorimétrico enzimático automatizado para as dosagens do TG, colesterol total (CT, VR $<200 \mathrm{mg}$ / dL) e HDL-colesterol (VR $<50 \mathrm{mg} / \mathrm{dL})$. A LDL-colesterol (VR $<130 \mathrm{mg} / \mathrm{dL}$ ) foi calculada a partir da fórmula de Friedewald. Os níveis de insulina (I, VR= 5,0-25 mUI/mL) foram avaliados pelo método de radioimunoensaio. A sensibilidade à insulina foi avaliada pelo método de HomaIR $(\mathrm{G}(\mathrm{mMol}) \times \mathrm{I}(\mathrm{i} \mathrm{U} / \mathrm{mL}) \div 22,5)^{11}$. Também calculamos a relação $\mathrm{G} / \mathrm{I}$, sendo considerado como sugestivo de RI quando menor do que $4,5^{12}$.

Segundo o National Cholesterol Education Program (NCEP) $)^{13}$, a SM foi definida como a presença de três ou mais dos seguintes fatores: obesidade abdominal (cintura $>88 \mathrm{~cm})$, intolerância à glicose $(\mathrm{G}$ de jejum $=110)$ ou DM2 $(\mathrm{G}$ de jejum $=126 \mathrm{mg} / \mathrm{dL}$ ou $\mathrm{Gpp}=200 \mathrm{mg} /$ $\left.\mathrm{dL}^{(14)}\right)$, hipertensão arterial (HA $=130 / 85 \mathrm{mmHg}$ ou uso de medicação), HDL baixo $(<50 \mathrm{mg} / \mathrm{dL})$ e hipertrigliceridemia $(T G=150 \mathrm{mg} / \mathrm{dL}$ ). O número de componentes da SM foi calculado. O RCV foi analisado utilizando-se como parâmetro o modelo preditivo de Framingham, que pontua, segundo tabela de escores de risco específica para sexo, as seguintes variáveis: idade, CT, HDL, PAS e PAD, tabagismo, DM2. O risco de desenvolver uma doença cardiovascular, em um período de 10 anos, foi estratificado segundo o escore de Framingham em: baixo (<10\%), moderado (entre 10 a $19 \%$ ) e alto $(=20 \%)$. A presença de aterosclerose manifestada clinicamente e a de DM2 já 
classificam o paciente como de alto $\mathrm{RCV}^{15}$.

Após análise dos dados, as pacientes foram divididas em dois grupos: com SM $(n=25)$ e sem SM $(\mathrm{n}=20)$. Para a análise estatística das variáveis numéricas, foram realizados os testes t de Student ou de Wilcoxon. Para as variáveis categóricas, foram utilizados os testes de Fisher ou c2 (Qui-quadrado). O coeficiente de correlação de Pearsonn e a regressão múltipla foram utilizados para identificação de associação entre o escore de RCV e as demais variáveis. Os resultados foram expressos em média e desvio-padrão. O valor de $\mathrm{p}<0,05$ foi considerado estatisticamente significativo.

\section{Resultados}

Nas 45 pacientes avaliadas, foi observado: DM2 em quatro (8,8\%), intolerância à glicose em 11 (24\%), HA em 20 (44\%), obesidade em 23 (51\%), hipertrigliceridemia em 26 (58\%), HDL baixo em 22 (48\%), obesidade central em $38(84 \%)$ e SM em 25 (56\%). Dez (22\%) pacientes apresentavam diagnóstico de SOP, sendo oito com SM.

A idade $(51 \pm 12 \times 36 \pm 13$ anos, $\mathrm{p}<0,001)$ e a cintura abdominal $(102 \pm 13 \times 90 \pm 13 \mathrm{~cm}, \mathrm{p}=0,005)$ foram significativamente maiores nas mulheres com SM, mas não houve diferença quanto ao IMC $\left(32,4 \pm 6,8 \times 29,4 \pm 6,8 \mathrm{Kg} / \mathrm{m}^{2}\right)$. Todas as pacientes com SM e 68\% daquelas sem SM apresentaram a cintura $>88 \mathrm{~cm}(\mathrm{p}<0,005)$. A PAS $(133 \pm 29 \mathrm{x}$ $116 \pm 18 \mathrm{mmHg}, \mathrm{p}=0,01)$ foi maior no grupo com SM do que no sem SM, enquanto a PAD $(83 \pm 22 \times 76 \pm 10 \mathrm{mmHg})$ não mostrou aumento significativo.

Os níveis de $G(106 \pm 30$ x $84 \pm 8,1 \mathrm{mg} / \mathrm{dL}$, $\mathrm{p}=0,0006), \operatorname{Gpp}(171 \pm 85 \times 97 \pm 20 \mathrm{mg} / \mathrm{dL}, \mathrm{p}=0,004) \mathrm{e}$ TG (212 $\pm 123 \times 98 \pm 65 \mathrm{mg} / \mathrm{dL}, \mathrm{p}=0,0001)$ foram maiores, enquanto o HDL-colesterol $(41 \pm 10 \times 53 \pm 11 \mathrm{mg} / \mathrm{dL}$, $\mathrm{p}=0,002)$ foi menor no grupo com SM. Não observamos diferença entre os grupos em relação aos níveis de CT $(223 \pm 37 \times 208 \pm 50 \mathrm{mg} / \mathrm{dL})$, LDL-colesterol $(132 \pm 33 \times$ $129 \pm 39 \mathrm{mg} / \mathrm{dL})$, insulina $(17,2 \pm 11,6 \times 12 \pm 8,8 \mathrm{mUI} / \mathrm{mL})$

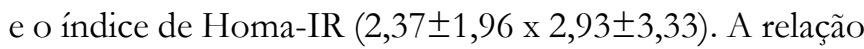
G/I foi compatível com RI em 10 casos (22\%), mas sem diferença estatística na proporção de casos entre os dois grupos $(7 \times 3, p=0,09)$.

O número de componentes da $\operatorname{SM}(4,6 \pm 1,2 \mathrm{x}$ 1,5 $\pm 0,8, \mathrm{p}<0,0001)$, o escore de risco de Framingham $(11 \pm 8,0 \times 2,4 \pm 2,5, \mathrm{p}<0,00001)$ (gráfico 1) e a proporção de casos com médio e alto risco $(56 \%$ x 0\%, p<0,0003) (gráfico 2) foram maiores no grupo com SM. O HOMAIR mostrou correlação linear com a cintura $(r=0,30$, $\mathrm{p}=0,04)$. O escore de risco mostrou correlação linear significativa com o número de componentes da SM $(\mathrm{r}=0,64, \mathrm{p}<0,001)$, cintura $(\mathrm{r}=0,41, \mathrm{p}=0,005), \mathrm{G}(\mathrm{r}=0,62$, $\mathrm{p}<0,001)$, PAS $(\mathrm{r}=0,47, \mathrm{p}=0,001)$ e HDL $(\mathrm{r}=-0,32$, $\mathrm{p}=0,04)$. Entretanto, no modelo de regressão múltipla, a $\mathrm{G}$ e a PAS foram variáveis independentes para o escore de risco $(\mathrm{r} 2=0,67, \mathrm{p}<0,007)($ tabela 1$)$. 
Tabela 1. Análise de regressão simples e múltipla do escore de risco de Framingham, tendo como variáveis independentes a glicose $(\mathrm{G})$, a pressão arterial sistólica (PAS), o HDL, a cintura abdominal e o número de componentes da síndrome metabólica ( $\mathrm{cSM})$ :

\begin{tabular}{|c|c|c|c|c|c|}
\hline \multirow[t]{2}{*}{ Variável dependente } & \multirow{2}{*}{\multicolumn{2}{|c|}{$\begin{array}{l}\text { Correlação simples } \\
(r)\end{array}$}} & \multicolumn{3}{|c|}{ Regressão múltipla } \\
\hline & & & $\beta$ & $S D$ & $r 2$ \\
\hline \multirow{5}{*}{$\begin{array}{l}\text { Escore de Risco } \\
\text { Cardiovascular }\end{array}$} & G & $0,72^{*}$ & 0,47 & 0,04 & 0,04 \\
\hline & PAS & $0,59 *$ & 0,34 & 0,04 & 0,04 \\
\hline & HDL-colesterol & $-0,31 * *$ & & & \\
\hline & Cintura & $0,45^{\#}$ & & & \\
\hline & $\mathrm{cSM}$ & $0,62^{*}$ & & & \\
\hline
\end{tabular}

G glicose de jejum; PAS pressão arterial sistólica; cSM componentes da síndrome metabólica

${ }^{*} \mathrm{p}=0,001,{ }^{* *} \mathrm{p}=0,04,{ }^{\#} \mathrm{p}=0,005,{ }^{\#} \mathrm{p}=<0,007$

Gráfico 1. Escore de risco de Framingham em mulheres com e sem síndrome metabólica (SM):

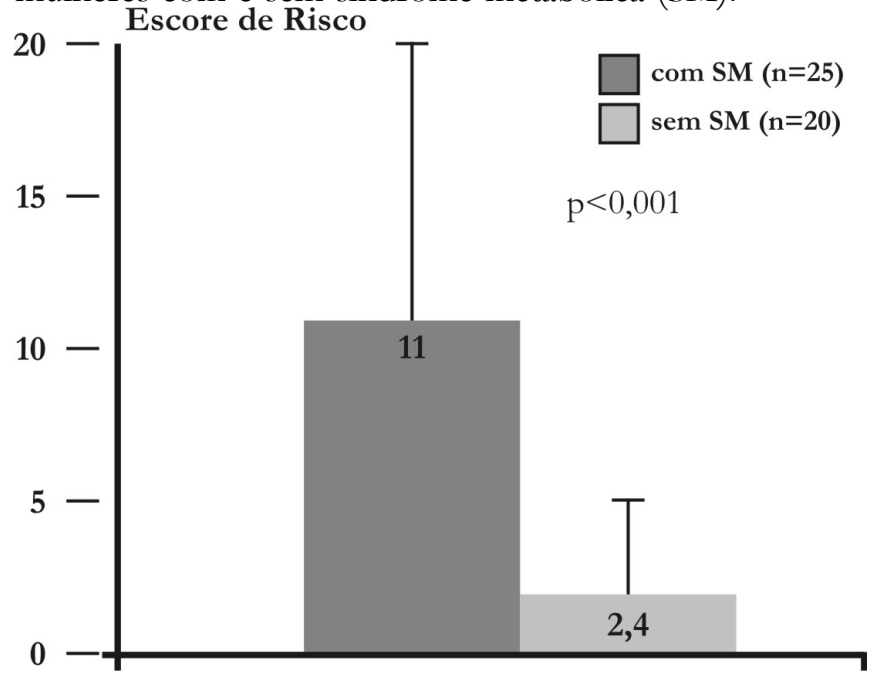

Gráfico 2. Proporção do risco cardiovascular baixo, médio e alto, segundo escore de risco de Framingham, em mulheres com e sem síndrome metabólica $(\mathrm{SM})$ :

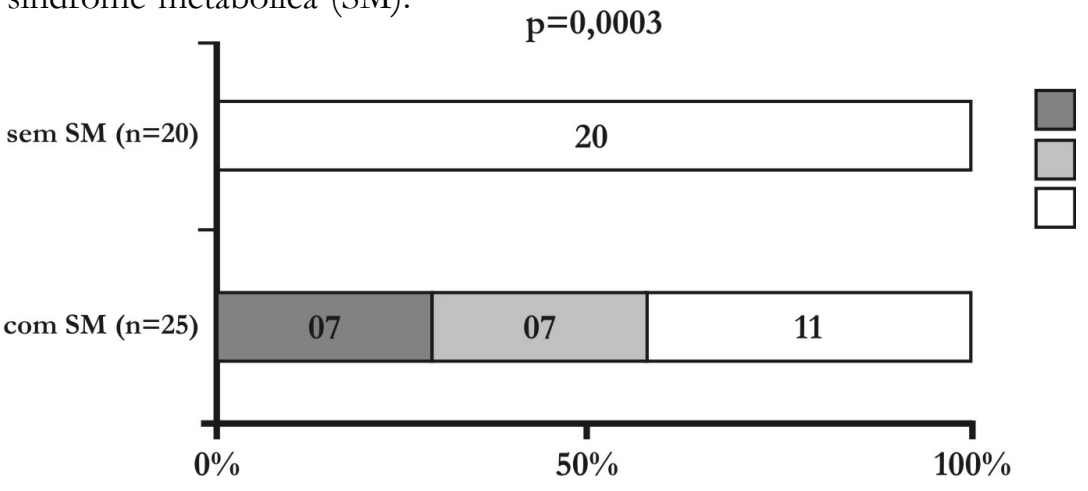




\section{Discussão}

A SM é um transtorno comum, que apresenta importantes implicações clínicas, porém, freqüentemente, não é detectada até uma fase tardia da vida, quando já ocorrem manifestações de DM2 ou complicações cardiovasculares. Contudo, nos últimos anos, tem aumentado às prevalências de obesidade e DM2, tanto na infância quanto em adultos, com evidente impacto global destas desordens. Essas tendências seculares podem sugerir que a obesidade e o DM2 - e por extensão a SM - irão tornar-se fatores etiológicos cada vez mais importantes de DCV no decorrer do tempo ${ }^{8,16}$. Logo, o diagnóstico da SM tornou-se uma importante ferramenta no dia-a-dia, por permitir avaliar, precocemente, o risco de desenvolvimento das desordens cardiometabólicas, e delinear estratégias terapêuticas específicas.

$\mathrm{Na}$ definição da SM existem algumas controvérsias, pois não há um único critério internacional com a descrição definitiva. A primeira definição da SM foi feita pela Organização Mundial de Saúde (OMS) ${ }^{17}$, que preconizou como ponto de partida a avaliação do distúrbio da glicose ou da medida de RI, o que impossibilitou a aplicação generalizada desse critério. A definição do NCEP-ATP III ${ }^{13}$ foi desenvolvida para o uso clínico e não exige a comprovação de RI, facilitando sua utilização. Pela sua simplicidade e praticidade é também a definição recomendada pela I Diretriz Brasileira de Diagnóstico e Tratamento da Síndrome Metabólica (I-DBSM $)^{10}$. Mais recentemente, a International Diabetes Federation ${ }^{18}$, com acadêmicos da OMS e do NCEP contribuindo para a tomada de decisão, propôs uma nova definição "unificada" que incluísse medida da cintura como requisito da SM. Além do aumento da cintura abdominal, o diagnóstico de SM pela IDF também exige a presença de pelo menos mais dois componentes - hiperglicemia de jejum $(>/=100 \mathrm{mg} / \mathrm{dL})$ ou DM2, hipertrigliceridemia, HDL baixo e HA. É interessante ressaltar que a IDF propôs pontos de cortes da medida da cintura inferiores (mulheres européias e sul americanas $>80 \mathrm{~cm}$ ), em comparação com os requisitos da NCEP, e etnicamente específicos, reconhecendo a maior sensibilidade aos efeitos metabólicos adver- sos do excesso de ganho de peso em vários grupos étnicos. A prevalência da SM depende em grande parte da definição empregada para sua identificação, mas também há diferenças relacionadas com o sexo, faixa etária, origem étnica e estilo de vida. De acordo com a OMS, sua prevalência varia de 1,6 a 15\%, dependendo da população e faixa etária estudada, sendo mais comum em mulheres. A prevalência de SM é muito baixa em crianças com peso normal, em torno de 3 a 8\%, mas aumenta paralelamente ao aumento da prevalência de obesidade, podendo ocorrer em até $30 \%$ de adolescentes obesos ${ }^{19}$. Segundo, Ford e cols, a prevalência aumenta com a idade, variando de $6,7 \%$ entre 20-29 anos até $43 \%$ após os 60 anos $^{20}$. Não há estudos sobre a prevalência da SM com dados representativos da população brasileira ${ }^{10}$. Neste estudo, as mulheres com SM apresentaram uma idade mais avançada, de acordo com outros estudos que mostram uma prevalência em torno de 50 a $60 \%$ na população acima dos $50 \operatorname{anos}^{10}$. Também observamos que a SOP é um achado comum em mulheres com SM. Recentemente, em mulheres com SOP a prevalência de SM foi descrita como de $46 \%$, sugerindo uma prevalência duas vezes maior do que a população geral ajustada para a idade e o sexo ${ }^{21}$.

A patogênese da SM é multifatorial. Os principais envolvidos são a RI e a obesidade. Vários fatores predispõem a síndrome como: inatividade física, idade avançada, disfunção endócrina e predisposição genética individual. O aumento da prevalência de obesidade no mundo e provavelmente da SM parecem resultar principalmente da obesidade exacerbada pelo estilo de vida sedentário ${ }^{8,13,17}$. Com base nos estudos populacionais, tem-se considerado que a obesidade é o fator que mais tem contribuído para a exacerbação da DCV no mundo industrializado. A cada quilo de peso adquirido, aumenta-se em 3,1\% o risco de coronariopatia. Sabe-se que a obesidade e a diminuição da atividade física aumentam-diversas vezes o risco de RI e, portanto, pioram a síndrome. Deve-se lembrar que esta síndrome pode ser encontrada em indivíduos sãos, com o peso e a tolerância à glicose normais ${ }^{22}$. Entretanto, têm sido descrito indivíduos de peso normal os quais são "metabolicamente 
obesos". Foi demonstrado que eles apresentam RI, porcentagem mais elevada de massa adiposa, particularmente de gordura visceral, e gasto energético mais baixo ${ }^{23}$.

Em nossas mulheres o IMC, o Homa-IR e os níveis de insulina foram semelhantes entre os dois grupos, talvez porque a maioria era obesa, mas mesmo assim houve diferença na cintura abdominal. Devemos ressaltar que o risco associado com a obesidade é bem identificado pelo aumento da cintura abdominal (obesidade abdominal ou visceral), enquanto a RI pode ser secundária tanto à obesidade como também a fatores genéticos ${ }^{8}$.

Todas as pacientes com SM apresentaram obesidade abdominal, mesmo aquelas com peso normal (IMC $<30 \mathrm{~kg} / \mathrm{m}^{2}$ ), e a cintura abdominal foi significativamente maior nessas mulheres, comparadas com aquelas sem a síndrome. Como a SM tem como ponto comum a RI e a obesidade abdominal ${ }^{3,4}, \log$, é correto presumirmos que o aumento da cintura é uma manifestação fenotípica clínica evidente de RI nessas pacientes. Além disso, observamos uma relação diretamente proporcional entre a cintura e o Homa-IR corroborando com essa idéia. Cada vez é maior a conscientização de que o acúmulo intra-abdominal de gorgordura, para o qual a medida da cintura é o melhor correlato antropométrico simples, relaciona-se fortemente com a RI, enquanto alterações da massa de gordura subcutânea têm relação menos forte. A gordura visceral também pode se relacionar melhor com outras vias de fatores de risco cardiovasculares ${ }^{24}$. Por isso, o aumento da cintura abdominal tem sido considerado como preditor da presença de RI, SM e conseqüentemente da DCV. Logo, a SM não é apenas um problema de peso, mas principalmente de cintura.

O mecanismo pelo qual a distribuição central da adiposidade causa RI já é bem conhecido. Depósitos viscerais de triglicérides possuem turnover mais acelerado que o de outras regiões, aumentando a oferta de ácidos graxos livres no sistema porta, estimulando a gliconeogênese e inibindo a depuração hepática da insulina, contribuindo para elevar a glicemia, a insulinemia e a RI ${ }^{25}$. A hiperin- sulinemia tem sido considerada uma condição de RCV independente e a obesidade abdominal é outra condição de risco ainda maior, independente do $\mathrm{IMC}^{8}$. Porém, o papel da RI e da hiperinsulinemia como fatores independen- tes do RCV ainda são controvertidos ${ }^{26}$.

Em pacientes com SM, o risco relativo de doença cardiovascular varia de 1,5 a 3,0 vezes, dependendo do estádio de progressão da síndrome ${ }^{4,8}$. O que tem sido questionado é se o aumento do RCV que está associado a SM é maior que o somatório dos fatores de risco. Nossas mulheres com SM apresentaram hiperglicemia, hipertrigiceridemia e redução de HDL, associados com níveis pressóricos mais elevados. Esses distúrbios agrupados, ligados pela RI, provavelmente conferiram um maior escore de risco a essas mulheres. Os componentes individuais da síndrome estão efetivamente estabelecidos como fatores de $\mathrm{RCV}^{16,18}$, assim, quando eles ocorrem simultaneamente, é lógico que deve haver maior chance de ocorrerem os resultados adversos. Estudos epidemiológicos fortemente sugerem que os múltiplos fatores de risco aumentam o risco mais que o somatório de simples fatores de risco, isto é, o risco aumenta geometricamente em vez de linearmente. Este fenômeno é chamado de risco multiplicativo ${ }^{8}$.

Além disso, parece que quanto maior o número de componentes da SM maior a chance de desenvolver $\mathrm{DM} 2^{27}$, e de acordo com os nossos dados também é maior o RCV, especialmente na presença de fatores de risco específicos como hiperglicemia e elevação da pressão arterial. A prevalência da DCV parece ser até três vezes maior em pacientes com SM, especialmente aqueles que já apresentam DM2. Embora nem todos os indivíduos com a síndrome desenvolvam DM2, é freqüente a síndrome culminar com DM2, que particularmente possui um alto risco para eventos cardiovasculares e outras complicações crônicas. Esta situação deve-se provavelmente ao fato de que, para este desenvolvimento é necessário um segundo componente fisiopatológico relacionado com um distúrbio na secreção de insulina, por parte das células beta ${ }^{1,3}$. O NCEP recomenda o tratamento de indivíduos com DM2 como sendo de "risco equivalente à doença coronariana". Esta recomendação reflete a evidência de que indivíduos com DM2 possuem risco de doença coronariana futura, 
da mesma forma que não-diabéticos com coronariopatia prévia. Da mesma forma, a SM é identificada como um marcador importante de RCV em indivíduos sem DM2, mas é considerada como um objetivo secundário da terapia, após a redução do $\mathrm{LDL}^{16}$. Tem sido sugerido que pacientes com DM2 ou com DCV clinicamente manifestada devam ser excluídos da definição da SM, visto que eles não fornecem nenhuma compreensão adicional de risco ou recomendações de tratamento que não são recomendados atualmente de outro modo ${ }^{28}$.

Nossas pacientes com SM apresentaram todos os graus de riscos fundamentados no modelo de Framingham, embora a maioria com severidade moderada à elevada. Como o risco de DCV é variável na síndrome e dependente da presença de fatores de risco específicos tem sido questionado o seu valor como um marcador de risco de $\mathrm{DCV}^{28}$. Além disso, na definição da SM não se considera outros fatores de risco igualmente relacionados à DCV, tais como idade, tabagismo, atividade física ou história de eventos prévios por DCV. Alguns desses e de outros fatores de risco fazem parte do algoritmo de Framingham, que tem mostrado, de modo geral, ser uma ferramenta mais potente na predição de eventos futuros de DCV a curto prazo. No entanto, mesmo a equação de risco de Framingham não inclui importantes fatores de risco (exemplo: obesidade, sedentarismo, história familiar, dentre outros) e mostrou ser pouco útil na predição de DCV em pessoas com DM $2^{29}$. A SM não parece ser um bom parâmetro de avaliação de risco global para DCV a curto prazo, logo a medida do risco em 10 anos pode ser avaliada melhor por meio de algoritmos, tais como o escore de Framingham. Entretanto, a síndrome é uma condição progressiva que começa com a resistência à insulina e obesidade visceral, piora com o avançar da idade e o aumento da obesidade. Por isso, devese ter em mente a importância da história clínica relacionada ao peso corporal quando da tentativa de estabelecer riscos, já que uma mulher obesa durante toda a sua vida pode apresentar aumento do risco de DCV em idade mais avança$\mathrm{da}$, enquanto que uma mulher que inicia sua obesidade em idade avançada pode não apresentar o mesmo risco ${ }^{2}$. Con- seqüentemente, a natureza progressiva da SM deve ser reconhecida, visto que os indivíduos com a síndrome têm uma longa trajetória de riscos a longo prazo tanto para DCV quanto para DM2. Quando DM2 e DCV existem concomitantemente, o risco para subseqüente morbidade cadiovascular é muito alta ${ }^{8}$.

A modificação no estilo de vida visa primordialmente à redução do peso, à prática de exercícios físicos com regularidade, ao maior consumo de fibras e ao menor consumo de gordura na dieta. No Finnish Diabetes Prevention Study, a mudança no estilo de vida reduziu em $58 \%$ o risco de DM2 nos pacientes com $\mathrm{SM}^{30}$. O Diabetes Prevention Program (DPP) demonstrou a intervenção intensiva no estilo de vida foi mais eficaz e segura (58\%) do que o uso de metformina $(31 \%)$ ou placebo na prevenção ou retardo do DM2 ${ }^{31}$. Até mesmo pequena redução do peso corpóreo $(5 \%$ a $10 \%)$ resulta em grande benefício cardiovascular com o controle da pressão arterial e das alterações metabólicas, além de representar uma meta a ser atingida durante o tratamento de pacientes com $\mathrm{SM}^{32}$. O sucesso das intervenções está estritamente relacionado com a modificação no estilo de vida, além do combate ao tabagismo, uso abusivo de álcool e estresse. Estas modificações irão melhorar a qualidade e a expectativa de vida das populações com SM. É claro que se poderia facilmente argumentar que, em lugar da ênfase sobre SM, o foco de atenção deveria ser o controle da obesidade abdominal e sua prevenção.

\section{Conclusões}

Neste estudo, as mulheres com SM apresentaram um maior risco cardiovascular que se mostrou diretamente relacionado com a cintura abdominal, o número de componentes e a presença de fatores de risco específicos. Acreditamos que como um conceito que denota o agrupamento de fatores de risco a SM tem sido um paradigma útil, pois na presença de uma das variáveis de risco num paciente deve incitar a busca para outras. Como o aumento da cintura pode ser considerado um bom marcador clínico de RI, sugerimos que a medida da cintura seja realizada rotineiramente, com o objetivo de tornar o rastrea- 
mento da SM um processo simples e eficaz de direcionar a conduta clínica. Considerando a elevada e crescente freqüência de obesidade nas populações, justifica-se o emprego de intervenções primárias dirigidas para a mudança no estilo de vida, de preferência desde a infância, com ênfase na perda de peso, realização de exercícios físicos e conseqüente redução da cintura abdominal, podendo prevenir a SM e reduzir a incidência de eventos cardiovasculares em mulheres adultas.

\section{Referências}

1- Sowers JR, Epstein M, Frohlich ED. Diabetes, Hypertension, and Cardiovascular Disease An Update. Hypertension 2001; 37: 1053-9.

2- Dickerson VM. Focus on primary care - Evaluation, management, and treatment of obesity in women. Obstetr Gynecol Surv 2001; 56: 650-63.

Sathyaprakash R, Henry RR. Prevenindo o Diabetes através do Tratamento dos Componentes da Síndrome Plurimetabólica. Current Diabetes Reports Latin América 2003; 2: 132-8.

4- Isomaa B, Almgren P, Tuomi T. Cardiovascular morbidity and mortality associated with the metabolic syndrome. Diabetes Care 2001; 24: 683-9.

5- Reaven GM. Role of insulin resistance in human disease. Diabetes 1988; 37: 1595-607.

6- Liese AD, Mayer-Davis EJ, Haffner SM. Development of the multiple metabolic syndrome: an epidemiologic perspective. Epidemiol Rev 1998; 20: 157-72.

7- Festa A, D'Agostino R, Howard G. Inflammation and microalbuminuria in nondiabetic and type 2 diabetic subjects: The insulin Resistance Atherosclerosis Study. Kidney International 2000; 58: 1703-10.

8- Grundy SM. Metabolic Syndrome: Connecting and Reconciling Cardiovascular and Diabetes Worlds. JACC 2006; 47: 1093-100.

9- Teixeira RJ and Simão Y. Insulin Resistance Syndrome including Obesity, Premature Pubarche and Polycystic Ovary Syndrome. In: Flamenbaum RK, editor Childhood Obesity and Health Research. New York: Nova Science Publisher;
2006. p 1-36.

10- I Diretriz Brasileira de Diagnóstico e Tratamento da Síndrome Metabólica. Arq Bras Card 2005; 84 (supl1): 128.

11- Mathews DR, Hosker JP, Rudenski AS, Naylor BA, Treacher DF, Turner RC. Homeostasis model assessment: insulin resistance and b-cell function from fasting plasma glucose and insulin concentrations in man. Diabetologia 1985; 28: 412-9.

12- Legro RS, Finegoo D and Dunaif A. A fasting glucose to insulin ratio is a useful measure of insulin sensitivity in women with polycystic ovary syndrome. J Clin Endocrinol Metab 1998; 83: 2694-7.

13- Third report of the National Cholesterol Education Program (NCEP) Expert Panel on the Detection, Evaluation, and Treatment of High Blood Cholesterol in Adults (Adult Treatment Panel III). Final Report (no authors listed). Circulation 2003; 106: 3143-420.

14- Sociedade Brasileira de Diabetes. Métodos e critérios para o diagnóstico de Diabetes Mellitus. In: Gomes MB, Dib SA, editores. Tratamento e acompanhamento de Diabetes mellitus Diretrizes da Sociedade Brasileira de Diabetes. Rio de Janeiro: Diagraphic; 2006. pp. 14-5.

15- Sociedade Brasileira de Cardiologia. IV Diretrizes Brasileira sobre Dislipidemias e Prevenção da Aterosclerose Departamento de Aterosclerose da Sociedade Brasileira de Cardiologia. Arq Bras Cardiol 2007; 88 (supl 1): 2-19.

16- Lindsay RS, Howard BV. Riscos Cardiovasculares Associados com a Síndrome Metabólica. Current Diabetes Reports - Latin América 2004, 3: 267-72.

17- Alberti KG, Zimmet P: Definition, diagnosis and classification of diabetes mellitus and its complications. Part 1: diagnosis and classification of diabetes mellitus provisional report of a WHO consultation. Diabet Med 1999, 15: 539-53.

18- Alberti KG, Zimmet P, Shaw J. The metabolic syndrome: a new worldwide definition. Lancet 2005; 366: 1059-62.

19- Cook S, Weitzman M, Auinger P, Nguyen M, Dietz WH. Prevalence of a metabolic syndrome phenotype in 
adolescents: findings from the third National Health and Nutrition Examination Survey, 1988-1994. Arch Pediatr Adolesc Med 2003;157: 821-7.

20- Ford ES, Giles WH, Dietz WH. Prevalence of the metabolic syndrome among US adults. Finding from the third national heath and nutrition examination survey. JAMA 2002; 287; 356-9.

21- Ehrmann DA. Polycystic Ovary Syndrome. N Engl J Med 2005; 352: 1223-36.

22- Zavaroni I, Bonora E, Pagliara M. Risk factors for coronary artery disease in healthy persons with hyperinsulinemia and normal glucosa tolerance. N Engl J Med 1989; 320:7026.

23- Dvorak Rv, CeNino Wf, Ades PA, Poehlmean ET. Phenotypic characteristics associated with insulin resistance in metabolically obese but normal-weight young women. Diabetes 1999; 48: 2210-4.

24-Matsuzawa Y. Therapy insight: adipocytokines in metabolic syndrome and related cardiovascular disease. Nat Clin Pract Cardiovasc Méd 2006; 3: 35-42.

25- Lerario DDG, Gimeno SG, Franco, LJ, Iunes M, Ferreira SRG. Prevalência de excesso de peso e implicações da distribuição abdominal de gordura para a síndrome metabólica em nipo-brasileiros. Rev Saúde Pública 2002; 36: 4-11.

26- Lindsay RS, Howard BV. Riscos Cardiovasculares Associados com a Síndrome Metabólica. Current Diabetes Reports - Latin América 2004, 3: 267-72.

27- Haffner SM. Carotid artery atherosclerosis in type2 diabetic and nondiabetic subjects with and without symptomatic coronary artery disease (The insulin resistance atherosclerosis Study). Am J Cardiol 2000; 85: 1395-400.

28-Kahn R, Buse J, Ferrannini E, Stern M. Síndrome metabólica: momento para uma avaliação crítica. Diabetes Care em português 2005; 4: 31-46.

29- Mc Ewan P, Williams JE, Griffiths JD, Bagust A, Peters JR, Hopkinson P, Currie CJ. Evaluation the performance of the Framingham risk equations in a population with diabetes. Diabetes Med 2004; 21: 318-23.

30- Tuomilehto J, Lindstrom J, Eriksson JG, for the Finnish

Diabetes Prevention Study Group. Prevention of type 2 diabetes mellitus by changes in lifestyle among subjects with impaired glucose tolerance. N Engl J Med 2001; 344: 134350.

31- The Diabetes Prevention Program Research Group: Reduction in the incidence of type 2 diabetes with lifestyle intervention or metformin. N Engl J Med 2002; 346: 393403.

32- Rosenbaum P, Ferreira SRG. An Update on Cardiovascular risk of Metabolic Syndrome. Arq Bras Endocrinol Metab 2003; 47: 220-7.

\section{Endereço para Correspondência:}

Rua Alfredo Pinto, 60/504

Tijuca - Rio de Janeiro - RJ

CEP: 20520-000

\section{Endereço eletrônico:}

rosijt@fst.com.br 